\title{
Recursos Humanos em Saúde no Fortalecimento da Atenção Primaria em direção a Saúde Universal: Prêmio APS Forte
}

Ana Paula Cavalcante de Oliveira; Mónica Padilla; Fernando Canto Micheletti; Denise Fernandes Leite

\section{RESUMO}

Este artigo analisa as experiências do Prêmio APS Forte relacionadas ao subtema Ampliação e flexibilização de horários identificando que os atributos do acesso/primeiro contato e da orientação comunitária embasaram a construção das experiências.

Palavras-chave: Atenção Primaria à Saúde; Recursos Humanos em Saúde; Política de Saúde; Sistema Único de Saúde; Estratégia de Saúde da Família.

\section{ABSTRACT}

This article analyzes the experiences of the APS Forte Award related to the subtheme Expansion and flexibility of schedules, identifying that the attributes of access / first contact and community orientation were the basis for the construction of experiences.

Keywords: Primary Health Care; Human Resources in Health; Health Policy; Health Unic System; Family Health Strategy.
Revista da Rede APS 2020

Publicada em: 04/09/2020

DOI:10.14295/aps.v2i3.148

Ana Paula Cavalcante de Oliveira

(Consultora Nacional em Recursos Humanos em Saúde na Organização Pan-Americana da Saúde/OMS, Brasil)

Mónica Padilla

(Coordenadora na Organização Pan-Americana da Saúde/ OMS, Brasil)

Fernando Canto Micheletti (Analista Técnico de Políticas Sociais, Ministério da Saúde, Brasil)

Denise Fernandes Leite (Consultora Técnica da Secretaria de Atenção Primária à Saúde do Ministério da Saúde, Brasil)

Correspondência para:

Ana Paula Cavalcante de Oliveira

cavalcaana@paho.org 


\section{INTRODUÇÃO}

Desde a criação do Sistema Único de Saúde (SUS) em 1988 e seguindo a constituição Federal em seu Artigo 196 1, o Brasil vem progressivamente mobilizando recursos para alcançar o acesso universal à saúde, tendo a força de trabalho em saúde um papel central na aceleração do alcance dos objetivos de saúde e no atendimento das necessidades da população em direção a Saúde Universal. O processo de implementação dos princípios do SUS exigia trabalhadores de saúde comprometidos com a mudança sistêmica no modelo de atenção, lastreada em reformas curriculares nas graduações em saúde. Também demandava uma estrutura hierarquizada e descentralizada de gestão capaz de absorver esta força de trabalho e, por ventura, atuar para corrigir percursos de formação debilmente alinhados com as diretrizes e políticas emanadas do SUS, utilizando frequentemente instrumentos de educação permanente e incentivos financeiros com base em indicadores de saúde. Se o comprometimento com a saúde universal por parte dos trabalhadores do setor, convertidos em competência e motivação para produzir cuidados de qualidade, foi o mote a que se recorreu a fim de reverter desigualdades no acesso à saúde, este anseio está ainda mais em voga na atualidade, justamente quando as necessidades assistenciais em saúde ganham contornos dramáticos com o alastramento da Covid-19 pelo território nacional.

Nesse contexto, a Atenção Primária à Saúde (APS) como primeiro nível de atenção é caracterizada por um conjunto de ações no âmbito individual e coletivo, com o objetivo de desenvolver uma atenção integral, orientada pelos princípios de universalidade, acessibilidade, continuidade do cuidado, integralidade da atenção, responsabilização, humanização e equidade (Brasil, S/A). Consolidando-se no SUS como um subsistema que deu vazão institucional a esse ímpeto

1 "A saúde é direito de todos e dever do Estado, garantido mediante políticas sociais e econômicas que visem à redução do risco de doença e de outros agravos e ao acesso universal e igualitário às ações $e$ reformista por meio de diretrizes, protocolos, programas e políticas que valorizaram a ação coordenada e colaborativa das equipes multiprofissionais em prol da atenção integral à saúde, mirando um horizonte de equidade no acesso. Reside aí sua potencialidade transformadora da práxis em saúde.

Ao longo dos últimos trinta anos vem sendo implementadas diversas estratégias no campo dos Recursos Humanos em Saúde (RHS) para a formação, fortalecimento da capacidade de gestão, educação permanente, aprimoramento das condições de trabalho, melhoria da qualidade no âmbito do SUS, em trajetória institucional que variou do âmbito local ao nacional ou vice-versa. Com a adoção destes programas e políticas em RHS juntamente à implementação da Estratégia Saúde da Família (ESF), considerada um mecanismo importante na ampliação do acesso e promoção da equidade em saúde, o Brasil atingiu uma cobertura pela ESF de $63,38 \%$ da população, correspondente a 132.137.891 de habitantes, através do aumento do número de equipes de saúde de 5.421 em 2001 para 42.784 em 2019 (Brasil, 2020b). Este salto permitiu um acesso inédito à saúde a uma população tradicionalmente alijada de serviços básicos, por falta de infraestrutura ou pelo não atendimento a critérios que vigoraram no passado, como a ausência de um vínculo empregatício formal.

Apesar dos importantes avanços realizados no país para disponibilizar e qualificar profissionais suficientes, bem preparados e melhor distribuídos, não há dúvida sobre as grandes dificuldades em responder às crescentes demandas de saúde. Entre os principais desafios da resposta ao mandato constitucional de saúde de implementar efetivamente o SUS proposto está um território nacional com grandes heterogeneidades, estruturas reguladoras frágeis em torno da formação e prática profissional em saúde, fragmentação dos diferentes níveis de gestão, fraca coordenação

serviços para sua promoção, proteção e recuperação." Brasil. Constituição da República Federal do Brasil. Art. 196, Distrito Federal: Senado Federal, 1988. 
dos processos de formação e integração na prática dos servidores.

Nesse contexto complexo, há avanços importantes que mostram ações bem-sucedidas realizadas pelas equipes de saúde nos diferentes níveis e espaços do SUS, que geram conhecimentos e práticas que, identificados e sistematizados, podem contribuir para o seu desenvolvimento. No marco da estratégia de Laboratórios de Inovação promovida pela Organização Pan-Americana da Saúde (OPAS) em parceria com o Ministério da Saúde (MS), no 25 ano de aniversário da ESF, foi lançado o "Prêmio Atenção Primaria à Saúde Forte para o SUS: Acesso Universal" com objetivo de "identificar, dar visibilidade, reconhecer e promover iniciativas locais, municipais ou regionais que tenham como foco a melhoria da APS" (MS e OPAS, 2019). Este trabalho visa analisar as experiências consideradas para o "Prêmio APS Forte" que contenham intervenções no campo da força de trabalho em saúde, realizadas para garantir o fortalecimento da APS no SUS. A análise foi realizada utilizando conceitos de disponibilidade, acessibilidade, aceitabilidade e qualidade dos profissionais de saúde propostos pela Aliança Global pela Força de Trabalho em Saúde e Organização Mundial da Saúde (OMS). Também visou-se relacionar as intervenções realizadas no âmbito da APS com as estratégias e políticas implementadas no país no campo da força de trabalho em saúde nos últimos trinta anos. Por fim, se propões a trazer reflexões quanto a importância do tema, aprendizados e potencialidade de escalonamento da implementação das intervenções realizadas por estas experiências na área de RHS a nível nacional.

\section{METODOLOGIA}

Trata-se de um estudo descritivo, com abordagem qualitativa. Utilizou-se de uma amostra de experiências consideradas admissíveis ao "Prêmio APS Forte" e documentos técnicos e científicos para dar suporte a análise. Com o intuito de caracterizar as experiências foi realizado uma análise temática, extraindo informações das experiências conforme categorias e subcategorias pré-determinadas na submissão ao prêmio e com a inclusão de outras a partir da leitura das experiências.

O quadro referencial utilizado para orientar a análise das experiências debruça-se em quatro dimensões aplicadas a força de trabalho em saúde: a disponibilidade (referindo-se por exemplo ao estoque adequado, produção e uma combinação de habilidades correspondentes a necessidade da população); acessibilidade (como a distribuição equitativas dos profissionais em termos de dimensões espaciais, temporal, e organizacional - buscando continuidade no atendimento entre os níveis de atenção); qualidade (avaliação dos profissionais de acordo com normas e percepção do usuário) e aceitabilidade (características e capacidades do profissional para tratar todos com dignidades, possibilitando a demanda por serviços através de provedores do mesmo sexo, mesma lingual e compreensão sociocultural entre outros) (Global Health Workforce Alliance e World Health Organization , 2013). Como uma mesma experiência analisada pode ser categorizada em diferentes dimensões, para efeito de análise, a experiência foi classificada na dimensão com maior expressão.

O Prêmio APS Forte teve um total de 1.239 experiências consideradas em sua $2^{a} \underline{a}$ fase de classificação - processo realizado em 4 fases (MS e OPAS, 2019). Para a composição da amostra de experiências analisadas neste trabalho, selecionamos as submetidas nas sete linhas temáticas consideradas no edital do Prêmio que foram classificadas nas categorias práticas de enfermagem (12 experiências) e RHS (20 experiências) e todas as submetidas na linha temática "estratégias de provisão e fixação de profissionais e estruturas em áreas remotas e/ou de vulnerabilidade, com ampliação do acesso" (30 experiências), compondo um total de 62 experiências. Também foi verificado, através de uma leitura rápida, se a categoria "processos de trabalho" (160 experiências) aplicava-se à área de RHS, que foram consideradas como pertencentes a temas da organização dos sistemas e serviços de saúde. 
Primeiramente, foi realizado uma leitura na íntegra das 62 experiências (distribuídas entre sete revisores) com aplicação dos critérios de inclusão e exclusão: se desenvolver no âmbito do fortalecimento da APS e apresentar intervenção na área de RHS, tendo o profissional como destaque da mesma. Para as experiências selecionadas foi realizado a análise temática. Numa segunda fase, todas as experiências foram analisadas objetivando verificar a exclusão das experiências que levantaram alguma dúvida acerca dos seu tema e/ou assegurar a consistência entre a classificação das experiências.

\section{RESULTADOS E DISCUSSÃO}

Após a aplicação dos critérios de inclusão e exclusão às 62 experiências submetidas ao
Prêmio foram selecionadas na primeira leitura 55 experiências e, após a segunda leitura, finalizou-se com um total de 53 experiências para análise (oito excluídas por não se referir a RHS e uma por não ser sobre APS).

\section{Caracterização geral das experiências}

De forma geral, a maior parte das experiências analisadas encontra-se em um estágio avançado de execução (65\%); sendo a maioria implementada no sul do país, seguida por sudeste e norte (30\%, 26\% e 25\%, respectivamente); e a grande maioria foi realizada a nível municipal (40\%) e a nível local (33\%), como Unidades de Saúde da Família (USF) e Unidades Básicas de Saúde (UBS), Gráfico 1 e 2 e Figura 1.

Gráfico 1 - Ano de início das experiências e estágio de execução

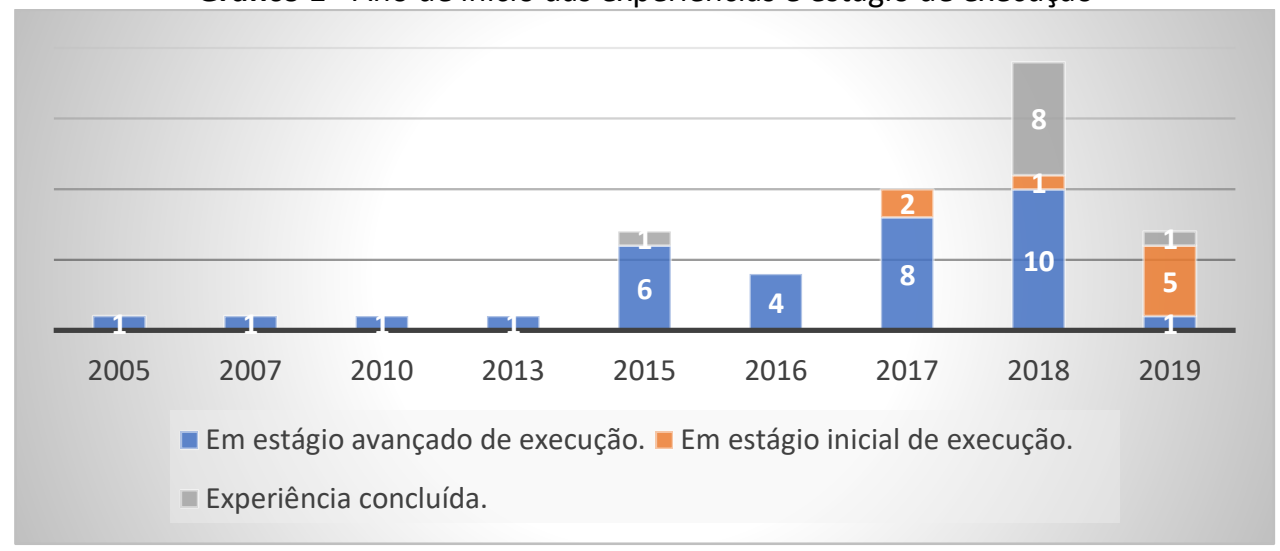

Fonte: elaboração própria com base nas experiências do Prêmio APS Forte em análise Nota: Uma experiência foi retirada do gráfico visto apresenta a data de início em 1905

Figura 1 - Número de experiências por Estado

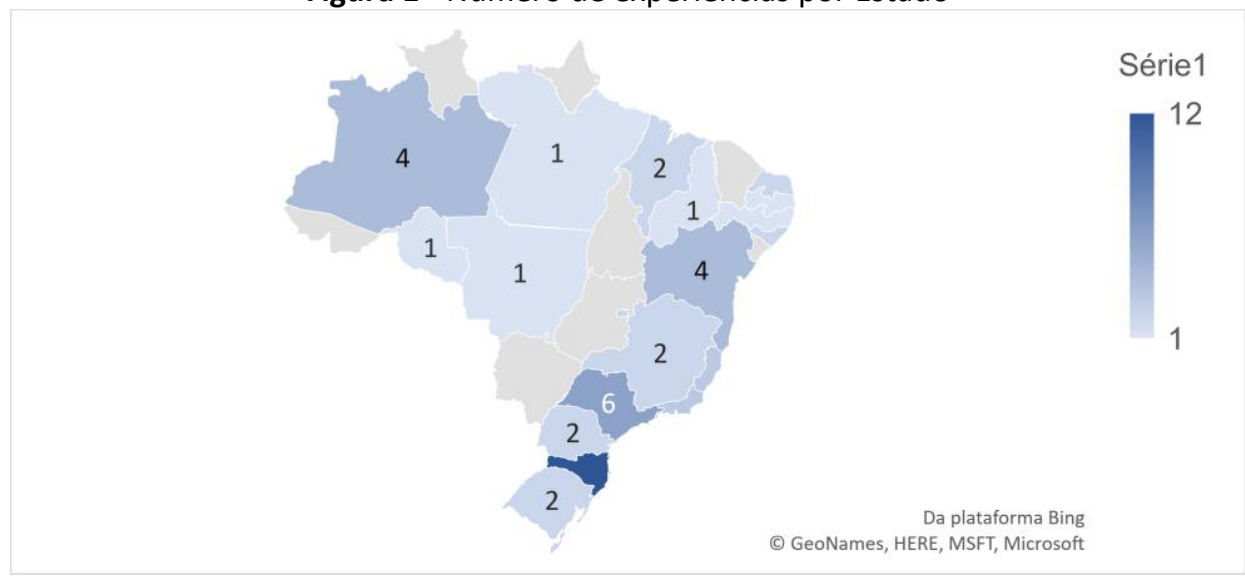

Fonte: elaboração própria com base nas experiências do Prêmio APS Forte em análise 
Gráfico 2 - Local de execução das experiências

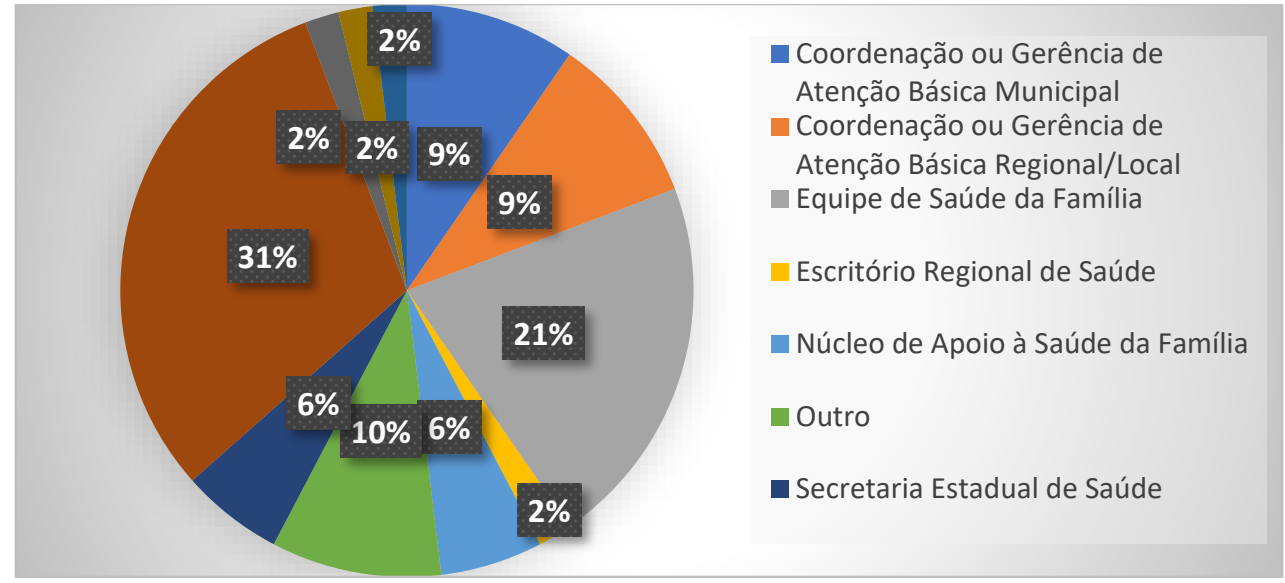

Fonte: elaboração própria com base nas experiências do Prêmio APS Forte em análise

Quanto ao profissional em foco na intervenção, a maioria das experiências apresentam em destaque o enfermeiro e, em seguida, a equipe multiprofissional (referente à experiência com mais de três profissionais em evidência), com $17 \%$ e $15 \%$, respectivamente. A visão de que a APS é uma área de atuação multiprofissional se reflete nas experiências, com 58\% indicando mais de um profissional como destaque da intervenção. Relativamente ao nível de qualificação destes profissionais, $52 \%$ das experiências referiam-se a profissionais de saúde de nível superior e $44 \%$ a experiências direcionadas aos de níveis superior e médio Tabela 1.

Tabela 1 - Profissional em destaque nas experiências

\begin{tabular}{|l|l|}
\hline Profissional em destaque na experiência & Número \\
\hline Agentes Comunitários de Saúde & 1 \\
\hline Assistente Social, Psicólogo e Visitadoras & 1 \\
\hline Enfermagem & 3 \\
\hline Enfermeiro & 9 \\
\hline Enfermeiro e Médico & 1 \\
\hline Equipe da saúde da família e do núcleo ampliado de saúde da família & 1 \\
\hline Equipe de saúde da família & 5 \\
\hline Equipe do núcleo ampliado de saúde da família e atenção básica (NASF-AB) & 1 \\
\hline Equipes de atenção primária & 3 \\
\hline Equipes de saúde bucal & 1 \\
\hline Farmacêutico & 1 \\
\hline Fisioterapeuta & 1 \\
\hline Médico & 3 \\
\hline Multiprofissional* & 8 \\
\hline Multiprofissional graduação e residência & 3 \\
\hline Nutricionista & 1 \\
\hline Odontologista & 1 \\
\hline Odontologista e Auxiliares em saúde bucal & 1 \\
\hline Residência de medicina de família e comunidade & 3 \\
\hline Residência de medicina de família e comunidade e graduação & 2 \\
\hline Residência multiprofissional em saúde da família & 2 \\
\hline Total & $\mathbf{5 2}$ \\
\hline Fonte: & \\
\hline
\end{tabular}

Fonte: elaboração própria com base nas experiências do Prêmio APS Forte em análise. 
ARTIGOS

ISSN 2596-3317 - DOI 10.14295/aps.v2i3.148

Oliveira, A. P. C.; Padilla, M.; Micheletti, F. C.; Leite, D. F.

Relativamente à intervenção executada na área de RHS de forma geral, $21 \%$ implementaram estratégias contendo ações em educação permanente/capacitação, $17 \%$ continham intervenções para a integração ensino-serviço, $15 \%$ com intervenções para a atração, fixação de profissionais em localidades ou área de cuidado com carência de profissionais (maioria destas utilizaram-se de medidas educacionais) e $15 \%$ das experiências continham uma combinação de intervenções para o alcance de seus objetivos Tabela 2.

\section{Dimensões das intervenções aplicadas a \\ força de trabalho em saúde na APS}

As dificuldades enfrentadas na acessibilidade ao profissional de saúde, principalmente geográfica, e inequidade na distribuição entre os níveis de cuidados é uma problemática recorrente no país. Cumpre ressaltar que este tópico foi objeto de atenção das autoridades desde antes da criação do SUS, com projetos pontuais que expandiram a oferta de serviços básicos de saúde, de hospitais de pequeno porte e a disponibilidade de RHS com vistas a ampliar o acesso da população aos cuidados de saúde. $O$ SUS alterou profundamente este cenário, ao tornar a saúde um direito universal e ao implicar os três entes federativos na responsabilidade pela provisão, financiamento e gestão da rede assistencial. Há uma linha, portanto, que separa estratégias como o Projeto Rondon (1968/89 e 2005) e Programa de Interiorização das Ações de Saúde e Saneamento (PIASS 1993/94), marginais ao sistema nacional de saúde então vigente, e as iniciativas que se sucederam a criação do SUS, desde o PSF, o Programa de Interiorização do Trabalho em Saúde (PITS 2001/04), Telessaúde (2007), Programa de Valorização dos Profissionais da Atenção Básica (PROVAB 2011/2017) e Programa Mais Médicos 2013 (OLIVEIRA et al., 2017), tanto em relação a sua escala e enquadramento institucional, já vinculados a políticas nacionais, quanto a seu ideário de integração ensino-serviço com o reconhecimento de que não há uma única diretriz capaz de enfrentar a escassez e má distribuição da força de trabalho em saúde no país. Ao eleger as iniquidades em saúde como alvo da ação governamental, o SUS necessita de estratégias com múltiplas frentes e ampla base de apoio para deixar seu caráter temporário e o risco de descontinuidade, sendo este um dos principais desafios no âmbito local, cuja permanência das iniciativas ainda depende em grande medida do suporte federal. Mas ao mesmo tempo que é improvável desvincular-se da agenda federal, há um amplo espaço para criatividade, dinamismo e inovações baseados no engajamento dos trabalhadores do setor e no apoio das lideranças locais.

Com $44 \%$ das experiências analisadas contendo intervenções implementadas para melhorar a acessibilidade aos profissionais é visível a persistência desta problemática no país, Tabela 2. A grande maioria das intervenções divide-se entre o emprego de estratégias para abordar a problemática de baixa atração de profissionais para a carreira na atenção primária, alta vacância nos processos seletivos, principalmente médicos, ou a dificuldade na fixação destes em áreas carenciadas ou de difícil acesso (aproximadamente $35 \%$ das experiências nesta dimensão); e a combinação de intervenções (com ações de educação permanente/capacitação em quase todas) para abordar diferentes problemas com o intuito de aumentar a resolutividade e dar continuidade no atendimento entre os níveis de atenção (aproximadamente 30\% das experiências nesta dimensão). As demais experiências utilizam de ações para a alocação dos profissionais (todas preocupadas com uma acessibilidade espacial às populações vulneráveis e rurais, atribuindo como causa da redução do acesso a falta de transportes, infraestrutura ou não abrangência da comunidade pelos serviços de saúde) e melhoria do processo de trabalho $(22 \%$ e $8 \%$ respectivamente).

As experiências analisadas que continham intervenções para a atração e fixação dos profissionais refletem o cenário das estratégias implementadas a nível nacional, sendo estas majoritariamente compostas por ações de educação por meio da mudança do conteúdo curricular, utilização das unidades da APS como campo de prática ou a exposição rural. Somente duas experiências utilizaram medidas 
combinadas com suporte pessoal/profissional, regulatória/administrativa e financeira (uma com a distinção de cargo para especialistas em medicina de família e comunidade - com salário diferenciado e função de liderança; e uma com a implementação de Plano de Qualificação e Avaliação da Atenção Básica), Tabela 3.

Tabela 2 - Intervenções por dimensão analisada e área de RHS

\begin{tabular}{|l|l|}
\hline Aceitabilidade & 3 \\
\hline Alocação & 1 \\
\hline Educação permanente/capacitação & 2 \\
\hline Acessibilidade & $\mathbf{2 3}$ \\
\hline Alocação & 5 \\
\hline Educação permanente/capacitação & 1 \\
\hline Processo de trabalho & 2 \\
\hline Alocação e Processo de trabalho & 1 \\
\hline Matriciamento e Educação permanente/capacitação & 2 \\
\hline Processo de trabalho e Educação permanente/capacitação & 2 \\
\hline Alocação e Educação permanente/capacitação & 2 \\
\hline $\begin{array}{l}\text { Estratégia de atração e fixação de profissionais para localidades ou área de cuidados com } \\
\text { carência de profissionais }\end{array}$ & 8 \\
\hline Disponibilidade & $\mathbf{1 2}$ \\
\hline Alocação & 1 \\
\hline Educação permanente/capacitação & 5 \\
\hline Processo de trabalho & 1 \\
\hline Ampliação do escopo de prática enfermagem (protocolo) & 5 \\
\hline Qualidade & $\mathbf{1 4}$ \\
\hline Acreditação (criação de indicadores para a enfermagem) & 1 \\
\hline Educação permanente/capacitação & 3 \\
\hline Integração ensino-serviço & 9 \\
\hline Avaliação da qualidade e Educação permanente/capacitação & 1 \\
\hline Total & $\mathbf{5 2}$ \\
\hline Fonte: laboraça propia com basenas experencias do Premio APS Forte & \\
\hline
\end{tabular}

Fonte: elaboração própria com base nas experiências do Prêmio APS Forte em análise

Respeito às estratégias para aumento da disponibilidade de profissionais de saúde, mesmo antes do impulsionamento dos RHS na agenda global (Relatório da OMS de 2006) e regional (O Chamado à Ação de Toronto para uma Década de RHS 2006/15), o Brasil tem desenvolvido o projeto de Profissionalização dos Trabalhadores da Área da Enfermagem (PROFAE 1999/03) para a expansão do treinamento em grande escala de auxiliares e técnicos de enfermagem; Programa de Formação de Profissionais de Nível Médio para a Saúde (PROFAPS 2009); Universidade Aberta do SUS (UNA-SUS 2010); Programa Mais Médicos 2013 (eixo da formação médica) (BRASIL, 2020a; OLIVEIRA et al., 2017) e; Programa Médicos pelo Brasil 2019.
Correspondem a esta dimensão $23 \%$ das experiências analisadas, que apontaram a existência de diversos desafios para manter a disponibilidade dos profissionais na APS. As intervenções propostas para vencer os desvios na prestação dos cuidados foram: o desenvolvimento de protocolos clínicos específicos para a ampliação do escopo de prática do enfermeiro com a prescrições de medicamentos e solicitações de exames objetivando aumentar a disponibilidade de determinados cuidados/serviços à população (42\% das experiências nesta dimensão); ações de educação permanente/capacitação - para desenvolver condições e capacidades dos profissionais e propiciar conhecimentos/habilidades para a melhoria da 
produtividade e disponibilização dos serviços (42\%); melhorias no processo de trabalho - para contribuir na redução de turnover de profissionais nos serviços (8\%) e; alocação dos profissionais - para a formação de uma equipe de saúde (8\%).

A estratégia de ampliar o papel dos enfermeiros vem sendo implementada em vários países por diferentes motivos como propiciar melhor equidade, aperfeiçoar o desempenho do setor, melhorar a qualidade e a relação custobenefício da prestação dos serviços e em resposta à escassez de categorias específicas de profissionais, sobretudo em áreas rurais ou remotas (CASSIANI e SILVA, 2019; TEMIDO e DUSSAULT, 2013). No Brasil, a revisão do escopo de prática tem ganhado importância crescente, sendo a ampliação da prática do enfermeiro implementada em alguns munícios e, no caso específico desta análise, todas as experiências que utilizaram desta estratégia são de um mesmo município. Para a sua implementação ampliada no Brasil, de forma geral, é imprescindível a interlocução dos diversos atores/setores (educação, serviços, regulação, população, outros profissionais) em seus diferentes níveis para definir a "ampliação do escopo de prática", estabelecer os requisitos formais para a atuação (qualificações, certificações, registro, etc.), os mecanismos regulatórios (CASSIANI e SILVA, 2019; KLEINPELL et al., 2014), as necessidades e prioridades dos serviços e formas de monitorização e avaliação da implementação.

As experiências submetidas ao Prêmio dirigidas prioritariamente a qualidade da força de trabalho em saúde representaram o segundo maior número de experiências nesta análise (com 14 experiências), utilizando intervenções no âmbito da integração ensino-serviço (64\% das experiências) e educação permanente/capacitação (22\% experiências). As experiências com intervenções na integração ensino-serviço contaram mais especificamente com a criação da Escola de Saúde Pública (ESP) objetivando a coordenação do processo de integração ensino-serviço-comunidade no âmbito da Secretaria Municipal de Saúde (SMS),
- PET-Saúde, e a utilização de Tecnologias Digitais de Informação e Comunicação (TDICS). Com a inserção dos discentes no serviço de saúde pode-se induzir novas formas de organização do trabalho para a melhoria do atendimento e resposta às necessidades de saúde da população; também por meio da troca de saberes (profissionais, discentes, docentes e usuários) favorecer na formação de um novo perfil profissional e na qualidade da prestação de seus serviços (BALDOINO e VERAS, 2016), onde a interprofissionalidade deve estar presente para garantir o acesso integral aos cuidados e serviços na atenção primária pelos usuários.

Diversas estratégias foram implementadas no Brasil visando à melhoria das condições que influenciam a qualidade dos profissionais de saúde referindo-se à regulação das instituições de ensino e treinamento e seus programas (como as Diretrizes Curriculares Nacionais 2001; o Sistema Nacional de Avaliação da Educação Superior - Sinaes 2004; e lista nacional de instituições credenciadas - cadastro e-MEC 2017); a transformação da formação dos profissionais (Política Nacional de Educação Permanente em Saúde-PNEPS 2004 e Programa para o Fortalecimento das Práticas de Educação Permanente em Saúde no SUS- PRO EPS-SUS 2018); a articulação ensino-serviço e comunidade (como o Programa de Educação para o Trabalho em Saúde - PET-Saúde 2010); e o fortalecimento da capacidade de gestão (Programa de Qualificação e Estruturação da Gestão do Trabalho e da Educação no SUS ProgeSUS 2006 e INOVA-SUS 2015). Quase todas as experiências analisadas, nesta dimensão, refletiram a mesma linha das estratégias implementadas a nível nacional, pois trouxeram intervenções para melhorar os fatores que influenciam "a qualidade dos profissionais de saúde", assim abordando a qualidade do profissional de forma indireta, com exceção da experiência que realiza a construção de indicadores objetivos para a enfermagem na APS presentes na acreditação municipal.

Por fim, observou-se que a aceitabilidade do profissional de saúde correspondeu a menor 
parte das experiências propostas ao Prêmio APS (três experiências). Mais concretamente duas continham intervenções no âmbito da educação permanente/capacitação e uma relativa à alocação de profissionais. Este fato espelha a representação que esta dimensão tem nas intervenções a nível nacional, no Brasil algumas estratégias foram implementadas dirigidas a aceitabilidade do profissional, como exemplo, o Programa Nacional de Reorientação da Formação Profissional em Saúde -PRO-SAÚDE 2005 (também classificado em acessibilidade e qualidade) e Programa de Incentivo às Mudanças Curriculares das Escolas Médicas PROMED 2002 (também abordando na dimensão qualidade) (CAMPBELL et al., 2013).

A fim de garantir que os profissionais de saúde sejam capazes de adaptarem suas respostas às diferentes características e valores culturais da população atendida e a se adaptarem a novos padrões da prestação de serviço, por exemplo, a telemedicina, o cuidado centrado no paciente e - autocuidado, são necessárias algumas competências e habilidades, como a sensibilidade cultural, empatia, capacidade de inovação e capacidade de atuar em equipes multidisciplinares, de forma interprofissional, e perante as novas tecnologias de comunicação e informação (DUSSAULT et al., 2018). Desta forma, existe a necessidade do desenvolvimento de intervenções visando diretamente a melhoria na aceitabilidade do profissional de saúde nos diferentes níveis de atenção, principalmente na APS. oO paradigma da culturalidade, que defende práticas como descentralização de serviços médicos e preservação de saberes locais, é uma revolução que se fez ainda mais necessária em tempos de pandemia do novo coronavírus (COLÓN e JENNINGS, 2020).

Reflexões da importância da problemática e implementação da intervenção de forma ampliada

No geral, as intervenções alcançam os objetivos propostos e geram resultados na expansão dos serviços, na melhoria da qualidade, na integração do trabalho das equipes, porém não foi possível identificar a criação de processos institucionais que garantam a sustentabilidade e que permitam ampliar a implementação das experiências analisadas. Percebe-se que a maior fortaleza das equipes está no desenvolvimento de processos de educação permanente e que os resultados da análise crítica dos processos de trabalho de maneira participativa constituem uma ferramenta eficaz para reorientar e obter melhoras substantivas no desempenho das unidades de saúde. Contudo, se observa que a sistematização das experiências deixa alguns vazios, pois requerem uma maior fundamentação de conceitos e utilização de indicadores que permitam caracterizá-las com maior precisão e projetá-las nas agendas dos gestores de saúde com maior claridade.

As intervenções descritas com maior potencial de institucionalização e de expansão em sua escala de implementação são a ampliação do escopo de prática do enfermeiro e a construção de indicadores objetivos para a enfermagem visando a acreditação de serviços como ferramenta de ordenação, afetando tanto a qualidade dos serviços como as quatro dimensões aplicadas a força de trabalho aqui analisadas, sem dúvida, um caminho de desenvolvimento necessário e desejável para melhorar o sistema de saúde em todos os níveis.

É necessário destacar a importância das residências como estratégia privilegiada para o recrutamento, seleção e fixação de profissionais de saúde, especialmente de médicos na especialidade de Medicina de Família e Comunidade e de outros profissionais da saúde via residência multiprofissional para a APS. As experiências de integração de ensino e serviço, associadas à formação de graduação e durante a residência aparecem igualmente como recursos chave de gestão para garantir uma força de trabalho qualificada e integrada, ainda que demande uma rede de preceptores para dar conta da atuação supervisionada desses profissionais.

Este trabalho apresenta algumas limitações como as inerentes à simplificação na classificação das estratégias em uma única dimensão, utilização dos conceitos macros em 
um nível micro e limitações trazidas pela característica da amostra a qual não permite generalizações.

\section{CONSIDERAÇÕES FINAIS}

O funcionamento eficaz dos sistemas de saúde requer uma força de trabalho suficiente e com a combinação de habilidades correspondentes a necessidade da população (disponibilidade), bem distribuída (acessibilidade), de qualidade (de acordo com normas e percepção do usuário), comprometida (aceitabilidade) e valorizada pelo sistema de saúde e pela população. Ter essa força de trabalho exige ações de políticas públicas consistentes, coerentes e sustentáveis, considerando a natureza intersetorial dos processos de planejamento e regulação da formação e do exercício profissional e o recrutamento, distribuição e desempenho dessa força, além da necessidade de resposta dinâmica às mudanças e demandas sociais, demográficas e epidemiológicas da população.

A formação de profissionais qualificados e comprometidos com a saúde de toda a população deu ímpeto à reforma sanitária nos anos 1980 e foi uma força motriz na engrenagem que levou o SUS ao patamar no qual se encontra, mobilizando esforços humanos e institucionais para torna-lo uma realidade viva na sociedade brasileira, da vacina nas UBS ao transplante de órgãos vitais, passando pela água que se consome às órteses e próteses desenvolvidas e o acesso a medicamentos; tecendo laços de solidariedade e empatia entre usuários e trabalhadores que foram institucionalizados em políticas de acolhimento e humanização que arejaram rígidas estruturas administrativas. Mas tem fôlego curto quando não estão associadas a políticas regulatórias que impactam no mercado de trabalho e garantam a disponibilidade e acessibilidade, o que contribui para a reprodução de históricas iniquidades em saúde.
O Brasil é, sem dúvida, um dos países com maior desenvolvimento na construção de políticas de RHS em busca da saúde universal, porém a implementação e principalmente a institucionalização de políticas nesse país de dimensão continental ainda exigem muitos esforços. Este trabalho, no contexto do Prêmio APS, permite reconhecer como as políticas públicas são construídas em nível local e como a sistematização dessas experiências pode ser uma maneira de criar sinergias e colocar não apenas problemas, mas também possíveis soluções na agenda política de aprimoramento do SUS.

\section{AgRAdeCIMENTOS}

Os autores agradecem a Alessandra Moreira (Consultora, Departamento de Gestão do Trabalho em Saúde), Cíntia Leal (Consultora, Departamento de Saúde da Família), Mirna Nóbrega (Consultora, Departamento de Gestão do Trabalho em Saúde) e Paulo Gomes (Departamento de Saúde da Família) pela contribuição na categorização das experiências.

\section{CONTRIBUIÇÕES}

APC Oliveira contribuiu com o desenho do estudo, coleta e análise dos dados, estruturação e escrita do artigo, e revisão da redação em todas as versões; $M$ Padilla contribuiu com o desenho do estudo, escrita do artigo e revisão da redação em todas as versões; FC Micheletti e DF Leite contribuiu com a coleta e análise dos dados, escrita do artigo e revisão da redação em todas as versões. 


\section{NOTAS E REFERÊNCIAS}

BRASIL. Ministério da Saúde. Secretaria de Atenção Primária à Saúde. O que é Atenção Primária? [Internet]. Ministério da Saúde, S/A. Disponível em: <https://aps.saude.gov.br/smp/smpoquee>_Acesso em 22 abr 2020.

BRASIL. Ministério da Saúde. Relatórios de Gestão [Internet]. Ministério da Saúde, 2020a. Disponível em: < Lwww.saude.gov.br/sgtes/relatorios-de-gestao>_Acesso em 4 abri 2020.

BRASIL. Ministério da Saúde. Sala de Apoio á Gestão Estratégica - Sage [Internet]. Indicadores de saúde a um clique. Brasília: Ministério da Saúde, 2020b. Disponível em: < http://sage.saude.gov.br/\# http://189.28.128.178/sage/> Acesso em 4 abr 2020.

BADOINO A; VERAS RM. Análise das atividades de integração ensino-serviço desenvolvidas nos cursos de saúde da Universidade Federal da Bahia Rev. esc. enferm. USP vol.50 no.spe São Paulo June 2016 https://doi.org/10.1590/S0080-623420160000300003

CAMPBELL J. et al. Human Resources for health and universal health coverage: fostering equity and effective coverage. Bull World Health Organ. 2013 Nov 1;91(11):853-63. doi: 10.2471/BLT.13.118729.

GLOBAL HEALTH WORKFORCE ALLIANCE, WORLD HEALTH ORGANIZATION. A universal truth: no health without a workforce [Internet]. Third Global Forum on Human Resources for Health Report, Recife, Brazil. Geneva: World Health Organization, 2013.

CASSIANI SHB; SILVA FAM. Ampliação do papel do enfermeiro na atenção primária à saúde: o caso do Brasil. Rev. Latino-Am. Enfermagem. 2019;27:e3245. DOI: https://doi.org/10.1590/15188345.0000.3245.

COLÓN M; JENNINGS E. Covid-19 mostra que medicina concentrada em grandes hospitais tem que ser superada. Folha de São Paulo Online. Disponível em: < www1.folha.uol.com.br/ilustrissima/2020/04/covid-19-mostra-que-medicina-concentrada-em-grandeshospitais-deve-ser-superada.shtml>. Acesso em 22 abr 2020.

DUSSAULT G. et al. Building the primary health care workforce of the 21st century - Background paper to the Global Conference on Primary Health Care: From Alma-Ata Towards Universal Health Coverage and the Sustainable Development Goals. Geneva: World Health Organization; 2018.

KLEINPELL R. et al. Addressing Issues Impacting Advanced Nursing Practice Worldwide. Online J Issues Nurs. 2014 May 31;19(2):5. 5 May 31, 2014. DOI: 10.3912/OJIN.Vol19No02Man05

MINISTÉRIO DA SAÚDE; ORGANIZAÇÃO PAN-AMERICAN DA SAÚDE. APS Forte para o SUS: Acesso Universal. Série técnica NavegadorSUS. Brasília, DF.: OPAS; 2019. Disponível em: < https://apsredes.org/wp-content/uploads/2019/10/BOOK-Pre\%CC\%82mio-Aps-Forte-site.pdf>. Acesso em 2 abr 2020.

OLIVEIRA APC de et al. Desafios para assegurar a disponibilidade e acessibilidade à assistência médica no Sistema Único de Saúde. Ciênc. saúde coletiva [online]. 2017, vol.22, n.4, pp.1165-1180. ISSN 1413-8123. https://doi.org/10.1590/1413-81232017224.31382016.

TEMIDO M; DUSSAULT G. How can a country learn from the experience of another? Expanding nurses' scope of practice in Portugal: Lessons from England. Health Policy 119 (2015) 475-487 http://dx.doi.org/10.1016/i.healthpol.2014.12.005 


\section{APÊNDICE}

Tabela 3. Experiências propostas ao Prêmio APS Forte na área de RHS por dimensão analisada

\begin{tabular}{|c|c|}
\hline Dimensão & Intervenções realizadas \\
\hline \multirow[t]{3}{*}{ Aceitabilidade } & $\begin{array}{l}\text { Ações de prevenção e promoção â saúde bucal dentro dos Centros Especializados em Reabilitações (usuários com deficiência) e encaminhamento a } \\
\text { UBS ou Centro de Especialidades Odontológicas (conforme a classificação de risco e o tipo de deficiência) }\end{array}$ \\
\hline & $\begin{array}{l}\text { Humanização no parto e compreender o indivíduo na sua localidade (acompanhamento da gestante pelos profissionais da casa de Apoio ao Índio e } \\
\text { levantamento de necessidades para a prestação de serviços mais humanizados e resolutivos) }\end{array}$ \\
\hline & $\begin{array}{l}\text { Realização de oficinas para profissionais de nível técnico e médio objetivando a sensibilização da importância do uso da inteligência emocional para } \\
\text { potencializar o desempenho, qualidade dos atendimentos (executores do primeiro contato com o usuário) e relações interpessoais colegas de } \\
\text { trabalho }\end{array}$ \\
\hline \multirow[t]{10}{*}{ Acessibilidade } & Campanha de imunização ao Influenza em áreas de difícil acesso \\
\hline & $\begin{array}{l}\text { Construção de um novo fluxograma para a organização do serviço de saúde no âmbito do cuidado com as feridas, criação de instrumentos próprios do } \\
\text { município para o acompanhamento do paciente e capacitação }\end{array}$ \\
\hline & Criação de programa de residência médica em medicina de família a fim de obter profissionais qualificados para atuação na APS no município \\
\hline & Guia de acolhimento com classificação de risco e avaliação de vulnerabilidades \\
\hline & $\begin{array}{l}\text { Implantação de um novo cargo para o profissional médico nas Equipes de Saúde da Família para profissional com formação técnica específica (com } \\
\text { diferencial salarial e para além de suas atribuições como médico tem como função a liderança) }\end{array}$ \\
\hline & $\begin{array}{l}\text { Integração da residência médica em medicina de família e comunidade com a graduação de medicina como estratégia de provisão e fixação de } \\
\text { profissionais na APS }\end{array}$ \\
\hline & Jornada de saúde bucal em ambiente escolar em uma comunidade rural \\
\hline & Projeto de educação, saúde e assistência social para a promoção de estimulação precoce para crianças em situação de vulnerabilidade \\
\hline & $\begin{array}{l}\text { Realização de treinamento de } 40 \text { horas do protocolo AIDPI para melhor acolhimento de demanda espontânea e encaminhamento responsável para } \\
\text { emergência pediátrica }\end{array}$ \\
\hline & $\begin{array}{l}\text { Ações itinerantes realizadas fora da UBS para garantia do acesso aos serviços de saúde e ações de educação e promoção da saúde à população } \\
\text { ribeirinha }\end{array}$ \\
\hline
\end{tabular}




\begin{tabular}{|c|c|}
\hline & Atendimento móvel facilitado para comunidades que residem distantes/rurais da UBS \\
\hline & $\begin{array}{l}\text { Atendimento de atenção farmacêutica em plantas medicinais e fitoterápicos e auriculoterapia com a inserção do ponto de atendimento do } \\
\text { profissional farmacêutico de práticas integrativas quinzenalmente na associação de moradores local }\end{array}$ \\
\hline & Organização de um fluxograma do serviço de nutrição para expor e apresentar a todas as equipes de saúde da atenção primária \\
\hline & $\begin{array}{l}\text { Introduzir uma atenção especializada de infectologista dentro da UBS do Sistema Prisional do estado: contrato de infectologista itinerante entre as } \\
\text { unidades prisionais; infectologista e equipe multidisciplinar a promover palestras e atividades de prevenção e conscientização sobre o HIV; coletadas } \\
\text { regularmente pela enfermeira da unidade prisional e; fornecimento da Terapia Antiretroviral (TARV), coleta e análise do CD4 e Carga Viral fornecidos } \\
\text { pelos Serviços de Atendimento Especializado (SAE) }\end{array}$ \\
\hline & Criação de um índice de vulnerabilidade das áreas de abrangência das UBS do município \\
\hline & $\begin{array}{l}\text { Desenvolvimento do programa de residência multiprofissional regionalizado em saúde da família, com o intuito de formar profissionais generalistas, } \\
\text { capazes de atuar em equipe, com atitudes colaborativas, no contexto da APS }\end{array}$ \\
\hline & $\begin{array}{l}\text { Residência em medicina de família e comunidade com estratégia para a fixação do profissional na área de trabalho e a continuidade da carreira como } \\
\text { médicos especialistas e preceptores }\end{array}$ \\
\hline & $\begin{array}{l}\text { Capacitar os médicos clínicos da APS para manejo das doenças reumáticas com a aplicação do protocolo de classificação da prioridade no } \\
\text { atendimento (matriciamento em reumatologia) e com o manejo do de baixa prioridade em suas unidades com apoio do especialista e da equipe } \\
\text { interdisciplinar }\end{array}$ \\
\hline & $\begin{array}{l}\text { Implantação de matriciamento do tratamento de feridas e lesões na ESF, com capacitações teórica e prática/em serviço e prestação de apoio matricial } \\
\text { presencial em visitas domiciliares e via WhatsApp do Referência Técnica (RT) municipal em feridas ao enfermeiro da equipe de ESF no atendimento ao } \\
\text { portador de feridas/lesões, em seu território adscrito }\end{array}$ \\
\hline & Plano de qualificação e avaliação da atenção básica instituído por lei municipal \\
\hline & $\begin{array}{l}\text { Oficinas de capacitação e inclusão dos auxiliares de enfermagem nas discussões sobre o processo de trabalho da UBS para potencializar a escuta mais } \\
\text { qualificada e resolutiva }\end{array}$ \\
\hline & $\begin{array}{l}\text { Acordo entre secretaria municipal de saúde e universidade para permite a vivência de "internato rural em medicina de família e comunidade" aos } \\
\text { alunos de graduação de medicina (estágio obrigatório na APS) e residência em medicina de família e comunidade (campo de estágio optativo), e } \\
\text { liberação dos profissionais médicos um dia por semana para ministrar as aulas na universidade e atividades de educação permanente }\end{array}$ \\
\hline & $\begin{array}{l}\text { UBS como campo de práticas para alunos de graduação (fisioterapia, medicina e terapia ocupacional) e residência médica em medicina de família e } \\
\text { comunidade para ampliação do acesso dos usuários ao cuidado e para a qualificação e fixação de profissionais }\end{array}$ \\
\hline Disponibilidade & $\begin{array}{l}\text { Construção dos protocolos de enfermagem na busca da qualificação de sua prática clínica visando a ampliação do acesso da população aos serviços } \\
\text { municipais de saúde }\end{array}$ \\
\hline & $\begin{array}{l}\text { Estruturar e normalizar a prática clínica da enfermagem a partir dos protocolos clínicos de enfermagem, a CIPE }{ }^{\circledR} \text { e guiar os Enfermeiros neste registro } \\
\text { formal }\end{array}$ \\
\hline
\end{tabular}




\begin{tabular}{|c|c|}
\hline & Inserção de DIU por enfermeiros com a elaboração de POP, capacitação dos profissionais e avaliação do impacto da ação \\
\hline & Projeto de capacitação de equipe técnica para desenvolvimento das atividades locais relativas ao programa bolsa família \\
\hline & Trabalho de sensibilização de equipe de saúde da família para ações preventivas e de promoção da saúde na UBS \\
\hline & $\begin{array}{l}\text { Realização de diagnostico (observação e questionário), utilização de ferramentas de gestão para realização da organização do processo de trabalho e } \\
\text { em proposta capacitação e implantação de Time de Resposta Rápida }\end{array}$ \\
\hline & $\begin{array}{l}\text { Educação permanente no âmbito do escritório regional de saúde: instituído um espaço de escuta para os servidores que estavam desmotivados e } \\
\text { também de estudo e reflexão sobre as relações e processos de trabalho, valorizando experiências para fortalecimento e compartilhamento de práticas } \\
\text { e sentimentos }\end{array}$ \\
\hline & $\begin{array}{l}\text { Realização de oficinas de "capacitação em educação permanente" com os profissionais da Atenção Básica do município, contando com quatro etapas: } \\
\text { diagnóstico situacional; produção de convites, material informativo e material audiovisual; realização das capacitações e; reuniões posteriores â cada } \\
\text { capacitação para análise e melhorados }\end{array}$ \\
\hline & $\begin{array}{l}\text { A inserção do diagnóstico para Leishmaniose, Tuberculose e Hanseníasena em UBS com atividades como capacitação dos profissionais da área de } \\
\text { diagnóstico para Hemoparasitas, supervisão, monitoramento do desempenho dos microscopistas e reuniões com secretário de saúde, coordenador da } \\
\text { atenção básica e gerente de endemias sobre a importância da descentralização para o diagnóstico dos três agravos nos municípios }\end{array}$ \\
\hline & Elaboração de protocolos e capacitação de enfermeiros da APS para enfrentamento da Sífilis \\
\hline & $\begin{array}{l}\text { Ampliar o acesso à saúde à população vulnerável de um conjunto habitacional e assegurar operacionalização de ações e serviços de saúde no âmbito } \\
\text { da APS, por meio da formação de uma equipe de saúde }\end{array}$ \\
\hline & $\begin{array}{l}\text { Estabelecimento de parceria entre a SMS e o Conselho Regional de Enfermagem para expansão dos protocolos clínicos de Enfermagem para } \\
\text { ampliação do acesso na APS (sensibilização e divulgação dos protocolos, instituição do Programa de Adesão aos Protocolos de Enfermagem na } \\
\text { Atenção Básica - atendimento de critérios mínimos e capação) }\end{array}$ \\
\hline Qualidade & $\begin{array}{l}\text { Criação da Escola de Saúde Pública (EAP) - objetivando a coordenação do processo de integração ensino- serviço-comunidade no âmbito da SMS, a } \\
\text { partir das necessidades sociais e do SUS, com ênfase na APS do município; e Programa Municipal de Bolsas de Estudo, Pesquisa e Extensão para a } \\
\text { Educação pelo Trabalho, sob gestão da SMS - objetivando possibilitar aos alunos a vivência prática do SUS }\end{array}$ \\
\hline & Descrever as práticas em saúde que integram ensino-serviço-comunidade que favorecem o cuidado integral aos usuários da USF \\
\hline & Encontros de educação permanente (mensais de quatro horas) para as equipes de saúde bucal utilizando metodologia de problematização \\
\hline & $\begin{array}{l}\text { Estudo exploratório retrospectivo dos principais indicadores de acesso e desempenho de uma unidade de atenção primária, com enfoque especial na } \\
\text { entrada da residência médica em medicina de família e comunidade e a descrição desses indicadores, entre } 2014 \text { e } 2015 \text {, respectivamente com a } \\
\text { ausência e a presença do programa de residência em medicina de família e comunidade da universidade do estado em uma das equipes da unidade }\end{array}$ \\
\hline & Oferta de curso sobre o uso racional dos medicamentos para os ACS \\
\hline
\end{tabular}




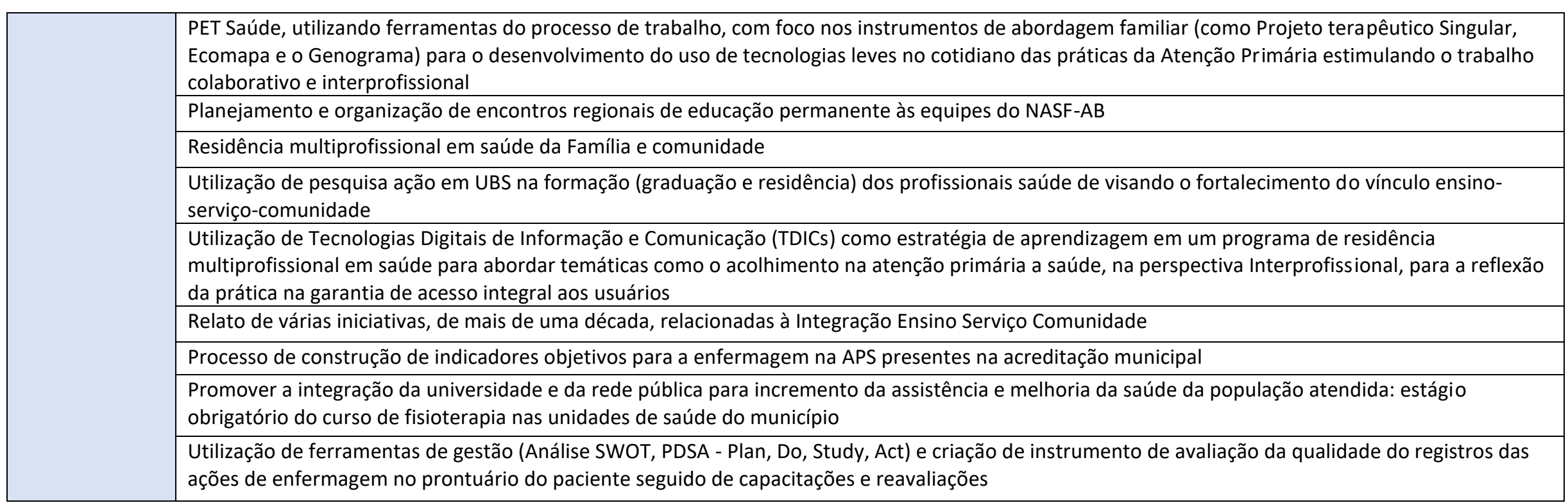

Fonte: elaboração própria com base nas experiências do Prêmio APS Forte em análise

Nota: UBS: Unidade Básica de Saúde; APS: atenção primária à saúde; ESF - Estratégia Saúde da Família; SMS: Secretaria Municipal de Saúde; USF: Unidade Saúde da

Família; NASF-AB: Núcleos Ampliados de Saúde da Família e Atenção Básica 